\title{
Análise comparativa de modelos conceituais de descrição arquivística: RiC e NEDA
}

\author{
Análisis comparativo de los modelos conceptuales de descripción archivística RiC y NEDA
}

Comparative analysis of the RiC and NEDA conceptual models for archival description

Marcos Vinicius BITtencouRt DE SouZA

\begin{abstract}
Marcos Vinicius Bittencourt de Souza, doctorando en Formación de la Sociedad del Conocimiento, Universidad de Salamanca (Spain), https://orcid.org/0000-0001-5292-7658, marcos.cpd@ufsm.br
\end{abstract}

\begin{abstract}
Resumen
El presente estudio analiza el modelo conceptual español para la descripción archivística (NEDA) y la nueva norma propuesta, Records in Context (RiC), buscando identificar puntos comunes y equivalencias entre sus entidades que puedan facilitar futuras migraciones de descripciones e intercambio de datos.
\end{abstract}

Palabras clave: Modelo conceptual. Descripción archivística Records in Context. NEDA-MC. Intercambio de datos.

\section{Introdução}

A década de 1990 foi de grandes avanços para a descrição arquivística graças à publicação da ISAD(G) (General International Standard Archival Description - Norma Geral Internacional de Descrição Arquivística) (ICA, 1994) pelo International Council on Archives (ICA - Conselho Internacional de Arquivos). As descrições dos arquivos tornaram-se mais uniformes facilitando e promovendo o intercâmbio entre as instituições custodiadoras. Subsequentemente à $\operatorname{ISAD}(\mathrm{G})$, o ICA criou três normas complementares, com objetivos e usos específicos: a) ISAAR(CPF) (International Standard Archival Authority Record for Corporate Bodies, Persons and Families - Norma Internacional de Registro de Autoridade Arquivística para Entidades Coletivas, Pessoas e Famílias) (ICA, 2003); b) ISDF (International Standard for Describing Functions - Norma Internacional para Descrição de Funções) (ICA, 2007), e; c) ISDIAH (International Standard for Describing Institutions with Archival Holdings - Norma Internacional para Descrição de Instituições com Acervo Arquivístico) (ICA, 2008). Após a publicação das normas, diversos países e regiões iniciaram esforços para a criação de normas específicas com o intuito de adaptá-las às suas realidades arquivísticas, como são os casos da NOBRADE (Norma Brasileira de Descrição Arquivística) (CONARQ, 2006), NORDA (Norma Dominicana de Descripción Archivística) (Archivo Nacional de la Nación, 2019a), NUDA (Norma Uruguaya de Descripción Archivística) (Archivo General de la

\begin{abstract}
The spanish conceptual model for archival description (NEDA) and the new proposed standard Records in Context (RiC) are analyzed, seeking to identify common points and equivalences among their entities that may help future migrations of descriptions and data interchange.
\end{abstract}

Keywords: Conceptual model. Archival description. Records in Context. NEDA-MC. Data interchange.

Nación, 2016b). Na Espanha foram desenvolvidos ainda padrões específicos para as suas regiões autônomas, de modo a abranger as suas especificidades, são elas: NOGADA (Norma Galega de Descrición Arquivística) (Grupo de Arquiveiros de Galícia, 2010) e NODAC (Norma de Descripción Archivística de Cataluña) (Generalitat de Cataluña, 2007). Todas essas normas citadas têm como pedra basilar a $\operatorname{ISAD}(\mathrm{G})$, utilizando os seus elementos e áreas de descrição fazendo as devidas adaptações de códigos e exemplos de aplicação individuais.

Na Espanha, em 2017, foi publicado o "Modelo Conceptual de Descripción Archivística" (NEDA$\mathrm{MC}$ ) visando a implantação de um novo padrão para a descrição arquivística espanhola, tendo como característica principal a descrição multidimensional. Em 2016, o Conselho Internacional de Arquivos publicou a primeira proposta de um novo padrão de descrição arquivística, nomeado "Records in Context" (ICA, 2016) que igualmente apresenta a característica de ser multidimensional.

O presente estudo analisa os padrões "Records in Context" e NEDA-MC procurando identificar intersecções de conceitos e aplicações assim como diferenças entre si, buscando apoiar futuros esforços de migrações entre os padrões. Primeiramente são apresentados os padrões internacionais em uso ISAD(G), ISAAR (CPF), ISDF e ISDIAH. Após são exploradas algumas das 
adaptações realizadas a níveis nacionais e regionais. Após, são apresentados os padrões NEDA-MC e "Records in Context", seguido pela análise comparativa entre os padrões e as conclusões encontradas no estudo.

\section{Normas ISAD(G), ISAAR(CPF), ISDF e ISDIAH}

A construção da primeira norma do ICA, a $\operatorname{ISAD}(G)$ trouxe uma mudança de paradigma no campo da descrição arquivística, mudando o foco anterior que era voltado principalmente à criação de instrumentos de acesso para a descrição do patrimônio arquivístico através de elementos de descrição. Essa mudança permitiu a criação de mecanismos que facilitam a gestão dos itens documentais sem prejudicar a localização e disponibilização do acervo documental (Lannes-Padrón, 2011, p.45).

A normalização da descrição arquivística, promovida pelo ICA, permitiu a interoperabilidade e facilitou a troca de informações entre diferentes arquivos. Como vantagem, as descrições podem ser realizadas uniformemente através de elementos padronizados, facilitando o acesso de usuários de arquivos e integrações de sistemas de informação.

A metodologia adotada nas normas do ICA foi a de descrição multinível unidimensional, configurando uma hierarquia entre os elementos a guiar a descrição do elemento mais genérico do arquivo até o item documental. Essa abordagem visa o reaproveitamento de informações de níveis superiores ao mesmo tempo em que, à medida em que a descrição avança no sentido dos documentos, detalhes específicos de cada nível vão sendo inseridos (ICA, 2000, p.12).

\section{NEDA-MC}

Em 2007, foi criada na Espanha a Comissão de Normas Espanholas de Descrição Arquivística (CNEDA - Comisión de Normas Españolas de Descripción Archivística) com o objetivo de assessorar a descrição arquivística espanhola desenvolvendo e atualizando as normas do país (CNEDA, 2017, p.6). O trabalho da comissão de imediato identificou a necessidade de atualizar a norma nacional iniciando o processo de elaboração que levaria à publicação, em 2017, do documento "Modelo Conceptual de Descripción Archivística: entidades, relaciones y atributos" (Modelo Conceitual de Descrição Arquivística: entidades, relações e atributos) (CNEDA, 2017).

$O$ modelo tem o intuito fornecer apoio à construção de novos padrões espanhóis de descrição arquivística, fornecendo um ponto de partida em comum

De maneira inovadora, o modelo apresenta a característica de focar em uma descrição multidimensional, não priorizando a hierarquização das entidades de arquivo, tal como acontece com a $\operatorname{ISAD}(G)$. A descrição não é mais focada somente nos documentos, mas traz à luz a necessidade de se descrever os contextos ao qual se inserem, mudando o paradigma estabelecido até então.

Conforme são identificadas as características do contexto, descrevem-se as entidades correspondentes relacionando-as aos documentos e demais elementos construindo uma rede de conexões que enriquece o arquivo. Além disso é favorecida a reutilização das entidades, permitindo que a atualização dos atributos de um determinado elemento do contexto sejam refletidas em todos os documentos os quais possui relação, melhorando a qualidade das informações (Zalama, 2018).

O modelo apresenta seis entidades principais que "constituem classes de objetos da realidade arquivística" (CNEDA, 2017, p. 9): a) Documentos de arquivo; b) Agente; c) Função e suas divisões; d) Norma; e) Conceito, objeto e acontecimento; e f) Lugar.

A entidade "Documentos de arquivo" foi subdividida em tipos que permitem acomodar a realidade presente nos arquivos. Aos agrupadores de documentos, foram reservados oito subtipos: a) Grupo de fundos; b) Fundo; c) Divisão de fundo ou de grupo de fundos; d) Séries; e) Subsérie; f) Fração de série ou de subsérie; h) Coleção; l) Divisão de coleção. A representação dos documentos em si e seus componentes é realizada através dos subtipos, respectivamente, Unidade documental e Componente documental.

A entidade "Documentos de arquivo" juntamente com os seus subtipos representam a granularidade dos níveis de agrupadores de documentos, partindo do nível mais genérico até o nível mais específico dos documentos.

A entidade "Agente" representa os agentes responsáveis por interagir com os elementos do arquivo através de eventos, tais como a produção do documento, gestão documental e a própria descrição arquivística. Essa entidade apresenta três subtipos: a) Instituição; b) Família; c) Pessoa. A entidade Agente apresenta uma estreita relação com a ISAAR(CPF) que prevê a descrição dos atores que interagem com o arquivo. A descrição de entidades custodiadoras de docu- 
mentos, função principal da norma ISDIAH, utiliza a entidade Instituição, um dos subtipos da entidade Agente.

Para preencher o papel da ISDF, no NEDA-MC foi adicionada a entidade "Função e suas divisões", que possui quatro subtipos: a) Função; b) Divisão de função; c) Atividade ou processo; d) Ação. As descrições que utilizam tais entidades permitem a identificação das ações efetuadas sobre os elementos do arquivo desde a gestão documental até as rotinas de preservação.

O modelo conta ainda com as entidades "Norma" que descreve os marcos legais que regem os documentos ou ainda as ações executadas pelos agentes, complementando o contexto dos documentos e respaldando os eventos realizados.

A entidade "Lugar" permite a descrição de uma localização geográfica, seja através da sua identificação exata com coordenadas de latitude e longitude, por exemplo, ou através da identificação de uma área, povoado, reinado, etc.

Por último, a entidade "Conceito, objeto y acontecimento" permite a descrição de entidades que não se encaixem no perfil das outras entidades definidas. Dessa forma, torna-se possível a adição de outras descrições que ajudam a enriquecer o contexto do arquivo, assim como auxiliam a descrição do conteúdo dos documentos.

\begin{tabular}{|c|c|c|c|}
\hline$I S A D(G)$ & $\begin{array}{l}\text { ISAAR(C } \\
P F)\end{array}$ & $I S D F$ & ISDIAH \\
\hline $\begin{array}{l}\text { Documentos } \\
\text { de Arquivo }\end{array}$ & Agente & $\begin{array}{l}\text { Funções e } \\
\text { suas } \\
\text { divisões }\end{array}$ & $\begin{array}{l}\text { Instituição } \\
\text { (subtipo de } \\
\text { Agente) }\end{array}$ \\
\hline $\begin{array}{l}\text { Grupo de } \\
\text { fundos, } \\
\text { Fundo e } \\
\text { Divisão de } \\
\text { fundos }\end{array}$ & $\begin{array}{l}\text { Instituiçã } \\
0\end{array}$ & $\begin{array}{l}\text { Função e } \\
\text { Divisão de } \\
\text { função }\end{array}$ & \\
\hline $\begin{array}{l}\text { Séries, } \\
\text { Subsérie e } \\
\text { Fração de } \\
\text { série ou de } \\
\text { subséries }\end{array}$ & Família & $\begin{array}{l}\text { Atividade ou } \\
\text { Processo }\end{array}$ & \\
\hline $\begin{array}{l}\text { Coleção e } \\
\text { Divisão de } \\
\text { coleção }\end{array}$ & Pessoa & & \\
\hline
\end{tabular}

Tabela I. Entidades NEDA-CM e relações com as normas ICA ( adaptado de CNEDA, 2017)

A Tabela I resume as relações das entidades NEDA-MC com as normas ICA.As entidades projetadas no modelo conceitual são consideradas obrigatórias e devem existir nos sistemas de descrição arquivística que implementem o modelo, mesmo que não existam descrições realizadas para cada uma. No entanto, o CNEDA deixa claro que podem ser criados tipos que se adaptem às realidades específicas de cada instituição custodiadora (CNEDA, 2017, p.10).

O modelo define, ainda, um conjunto de relações possíveis entre as entidades existentes. Para o CNEDA (2017, p.21), as relações são partes fundamentais do modelo, uma vez que "[...] preservam o significado, o valor testemunhal, o contexto e a acessibilidade dos documentos do arquivo através do tempo". São previstas relações sob sete perspectivas diferentes: 1) Contexto e valor testemunhal dos documentos; 2) Estrutura dos documentos; 3 ) Conteúdo dos documentos e valor informativo; 4) Associação entre documentos; 5) Vinculação entre agentes; 6) Estrutura das funções, e; 7) Associação entre funções. O NEDA-MC apresenta ainda os atributos das entidades e das relações que "[...] representam características informativas que se consideram de utilidade para o conhecimento de tais entidades e relações" (CNEDA, 2017, p.48, tradução nossa). De forma a esclarecer os usos dos atributos, o modelo detalha as suas utilizações, obrigatoriedades e restrições de uso, adicionalmente.

Um ano antes da publicação do modelo conceitual espanhol, o Conselho Internacional de Arquivos publicou a sua nova proposta de norma para descrição arquivística, nomeada Records in Context (RiC). Embora os modelos sejam contemporâneos e as suas comissões de elaboração possuam membros em comum, o resultado de cada órgão mostrou-se distinto, apresentando entidades, atributos e relações diferentes. Mesmo diferentes, ambos compartilham o conceito central da multidimensionalidade das descrições.

\section{Records in Context (RiC)}

Em 2012, o ICA constituiu o Grupo de Especialistas em Descrição arquivística (Experts Group on Archival Description - EGAD) e a ele atribuiu a missão de desenvolver o novo padrão internacional de descrição arquivística. O trabalho foi reconhecido pelo próprio EGAD como um "desafio assustador" (ICA, 2016 p.1) à medida em que visa contemplar a realidade heterogênea dos arquivos mundiais.

O processo foi conduzido de forma colaborativa recebendo as contribuições da comunidade arquivística mundial. A primeira proposta elaborada pelo EGAD foi exposta no documento "Records in Context: A Conceptual Model for Archival Description. Consultation Draft v0.1" ("Records in Context: Modelo Conceitual para Descrição Arquivística. Rascunho de consulta versão 0.1.”) 
(ICA, 2016). Como o próprio documento esclarece, é apresentado um rascunho a ser estudado e analisado por arquivistas e demais profissionais envolvidos na descrição arquivística a fim de gerar um entendimento comum que embase a finalização do modelo conceitual.

A ideia principal e norteadora do modelo é relacionar o documentos ao seus contextos criando uma descrição rica em elementos que expressa a sua proveniência (Llanés-Padron; Moro-Cabero, 2017). Ao se descrever um documento, de pronto são identificados dados externos a ele, pertencentes ao contexto o qual está emergido, fazendo com que seja necessária a descrição dos outros elementos (Zalama, 2018, p.33). Tais descrições devem se tornar independentes, porém relacionadas entre si aprimorando o contexto geral do acervo arquivístico.

$\mathrm{Na}$ realidade atual, dos documentos digitais e da pós-custodialismo, em que muitas vezes os documentos são elaborados e produzidos colaborativamente, o $\mathrm{RiC}$ busca dilatar o conceito original da proveniência, permitindo que os documentos descritos pertençam a mais de um fundo, por exemplo, graças aos relacionamentos previstos no modelo (ICA, 2016). Segundo Llanés-Padrón e Moro-Cabero (2017), o RiC se adapta aos novos tempos e contribui na gestão documental, preservação de documentos e reutilização de dados através da sua maneira como prevê a descrição dos contextos.

Após a primeira rodada de recebimento de contribuições da comunidade arquivística, o EGAD realizou um intenso trabalho para efetivar as alterações sugeridas e, em dezembro de 2019, publicou a primeira versão da ontologia referente ao RiC (RiC-O) (ICA, 2019b) e a prévia da segunda versão do modelo conceitual (ICA, 2019a).

Mesmo que o caráter do documento seja de versão prévia, o ICA elucida que ele já substitui a versão 0.1 anterior (ICA, 2016) e, embora não seja o documento completo do modelo conceitual, "[...] inclui as principais seções do RiC-CM: entidades, seus atributos, e relações que podem existir entre elas" (ICA, 2019c, tradução nossa). A publicação visa apoiar a compreensão da versão v0.1 da ontologia RiC-O "[...] provendo contexto para as classes, propriedades e apresentando a lógica geral da RiC-O [...]" (ICA, 2019c, tradução nossa). A finalização da versão 0.2 do modelo conceitual está prevista para ser disponibilizada para o ano de 2020 adicionando exemplos de uso, definições mais acuradas e documentações de apoio.

O projeto de entidades do RiC-CM foi realizado através da hierarquia de entidades, utilizando conceitos bem estabelecidos e concretizados no paradigma de orientação a objetos. Essa metodologia permite que as entidades herdem as propriedades e comportamentos das classes superiores, facilitando a manutenção e evitando a repetição de informações (Snyder, 1986). Em alguns casos, o modelo apresenta até quatro níveis de herança das entidades.

\begin{tabular}{l}
\hline - RiC-E01 Thing \\
\hline - RiC-E02 Record Resource \\
\hline - RiC-E03 Record Set \\
\hline - RiC-E04 Record \\
\hline - RiC-E05 Record Part \\
\hline - RiC-E06 Instantiation \\
\hline - RiC-E07 Agent \\
\hline - RiC-E09 Group \\
\hline - RiC-E10 Family \\
\hline - RiC-E11 Corporate Body \\
\hline - RiC-E12 Position \\
\hline - RiC-E13 Mechanism \\
\hline RiC-E14 Event \\
\hline - RiC-E15 Activity \\
\hline RiC-E16 Rule \\
\hline - RiC-E17 Mandate \\
\hline RiC-E18 Date \\
\hline - RiC-E19 Single Date \\
\hline - RiC-E20 Date Range \\
\hline - RiC-E21 Date Set \\
\hline
\end{tabular}

Tabela II. Entidades do modelo RiC (elaborado a partir de ICA, 2019a)

O modelo apresenta 22 entidades, sendo a entidade RiC-E01 Thing, a mais genérica e considerada a raiz de todas. Nela são definidas propriedades e comportamentos que são herdados por todas as outras entidades. Por seu caráter mais genérico, o seu uso deve se restringir aos casos em que as demais entidades não consigam representar o conceito a ser descrito tais como conceitos abstratos e movimentos culturais, por exemplo (ICA, 2019). A Tabela II exibe os tipos de 
entidades presentes no RiC, sendo possível notar que todas descendem da entidade RiC-E01 Thing e que a entidade RiC-E07 Agent possui dois níveis de subtipos associados à ela.

A entidade RiC-E02 Record Resource possui três subtipos: a) RiC-E03 Record Set: representa os agrupadores de documentos geralmente relacionados ao Plano de Classificação de Documentos da instituição (PCD), como fundos, coleções ou séries; b) RiC-E04 Record: representa o documento arquivístico a ser descrito; c) RiC-E05 Record Part: se refere a uma parte integrante de um documento, sem o qual não pode existir separadamente, estando sempre atrelada ao documento ao qual pertence.

A entidade RiC-E06 Instantiation é herdeira direta de RiC-E01 Thing e não possui subtipos. O seu uso deve ser realizado para descrever as formas de representações dos documentos arquivísticos. Para exemplificar o seu uso, pode-se pensar na descrição de um documento digitalizado, com o seu original em papel, descrito com a entidade RiC-E04 Record. Nesse caso, devese criar duas instâncias da entidade RiC-E06 Instantiation para descrever as características específicas da representação original em papel e para a descrição das características da representação digital. Cada uma das representações possuirá informações diferentes contendo os detalhes dos seus suportes, por exemplo.

A entidade RiC-E07 Agent tem o papel de registrar a descrição das figuras que interagem ou estão relacionadas aos elementos do arquivo, como arquivistas, autores, produtores, etc. A entidade possui quatro subtipos: 1) RiC-E08 Person: descreve seres humanos ou uma personalidade existente que interage com o arquivo; 2) RiC-E09 Group: representa grupo de agentes reconhecido no contexto social; 3) RiC-E12 Position: descreve um cargo ocupado por um agente e que existe independente da existência do agente, como, por exemplo, presidente da república; 4) RiC-E13 Mechanism: utilizado para descrever um sistema ou software que, por exemplo, produziu um determinado documento.

A entidade RIC-E09 Group ainda se subdivide em dois tipos: 1) RiC-E10 Family: utilizado para descrever uma família, um relacionamento social reconhecido como família ou, até mesmo, uma dinastia; 2) RiC-E11 Corporate Body: representa um determinado órgão, entidade ou instituição que se relaciona com as demais entidades do modelo.

Os acontecimentos presentes no arquivo são descritos com a entidade RiC-E14 Event, registrando, por exemplo, a produção de um documento, verificações de integridade e tramitações de um processo. A entidade possui o subtipo RiC-E15 Activity que está ligada às atividades que as pessoas desenvolvem para a realização de um determinado processo.

A entidade RiC-E16 Rule rege as normas que embasa a atuação dos agentes no desenvolver de suas atividades em interação com os elementos do arquivo. A entidade RiC-E17 Mandate é um subtipo de RiC-E16 Rule sendo usada para representar explicitamente leis, mandatos ou, até mesmo, um plebiscito que resguardam as ações tomadas por agentes.

A representação de datas e períodos de importância para o arquivo é realizada através da entidade RiC-E18 Date e seus subtipos: a) RiCE19 Single Date: utilizada para um instante único do tempo, como uma data histórica; b) RiC-E20 Date Range: delimita um período relacionado aos documentos como, por exemplo, uma década ou um semestre acadêmico; c) RiC-E21 Date Set: representa um conjunto de vários tempos distintos, empregada, por exemplo, para descrever as faixas temporais de documentos contidos em um RiC-E03 Record Set. A exatidão das datas utilizadas na tarefa de descrição nem sempre é possível de ser verificada, sendo muitas vezes aproximada. Para sinalizar esse grau de incerteza, as entidades que representam as datas possuem o atributo RiC-A26 Certainty.

Os lugares físicos pertinentes à descrição devem ser representados através da entidade RiC-E22 Place, podendo representar uma localização geográfica bem delimitada com coordenadas de latitude e longitude, como também para descrever uma região ou um povoado constituído.

A versão 0.2 do RiC (ICA, 2019) prevê 78 tipos de relações entre as entidades do modelo, representando uma redução drástica nessa quantidade que, na versão 0.1 era de 792 (ICA, 2016). $\mathrm{O}$ alto número de relações da versão anterior foi alvo de sérias críticas da comunidade arquivística, pois tornaria o modelo burocrático, de difícil entendimento e implementação nos sistemas de descrição (Conselho Nacional de Arquivos, 2016; Gillean, 2017; Interpares Trust, 2016).

A forma como as informações são informadas em cada entidade e/ou relação são apresentadas como atributos, totalizando 41 atributos de entidades e 5 atributos de relações de entidades.

$O$ uso dos relacionamentos entre as entidades do arquivo auxilia a descrever o contexto de produção e gerenciamento dos documentos assumindo um alto grau de importância no registro das ações realizadas durante a história dos documentos (ICA, 2019). Dessa forma, a descrição arquivística deve procurar identificar o máximo 
de ligações possíveis entre os elementos a fim de que se conheçam mais detalhes sobre o contexto dos documentos, incrementando inclusive o grau de autenticidade dos elementos descritos.

Da mesma forma como as entidades, as relações foram elaboradas através de hierarquia, sendo a relação "RiC-R001 is related to" a relação raiz e mais genérica, da qual todas as demais descendem.

\section{Mapeamento das entidades entre os modelos conceituais}

A fim de auxiliar a migração das descrições realizadas em um modelo para outro modelo, são apresentadas as correlações e entre as entidades dos modelos. Pode-se inferir que a entidade "Documentos de arquivo" do modelo NEDA corresponde à entidade RiC-E02 Record Resource.

A Tabela III exibe a correspondência dos subtipos da entidade NEDA "Documentos de arquivo" para as entidades do RiC. É possível notar que oito subtipos da entidade NEDA correspondem à uma entidade do RiC. Dessa forma, a descrição de todos os agrupamentos de documentos do NEDA pode ser realizada com o uso da entidade RiC-E03 Record Set, que possui o atributo RiCA36 Record Set Type para diferenciar o tipo de agrupamento que está sendo descrito, caso necessário. Esse atributo equivale também à propriedade "3.1.4 Level of description" da ISAD(G) que "identifica o nível de arranjo da unidade de descrição" (ICA, 2000, p.16, tradução nossa).

\begin{tabular}{ll}
\hline Entidade NEDA & Entidade RiC \\
\cline { 1 - 1 } Documento de arquivo & RiC-E02 Record Resource \\
\cline { 1 - 1 } Grupo de fundos & RiC-E03 Record Set \\
\cline { 1 - 1 } $\begin{array}{l}\text { Fundo } \\
\text { Divisão de fundos/ Grupo }\end{array}$ & \\
\cline { 1 - 1 } Série & \\
\cline { 1 - 2 } Subsérie & \\
\hline Fração de série/ Subsérie & \\
\cline { 1 - 2 } Coleção & RiC-E04 Record \\
\hline Divisão de coleção & RiC-E05 Record Part \\
\hline Unidade documental & \\
\cline { 1 - 2 } Componente documental & \\
\hline
\end{tabular}

Tabela III. Mapeamentos das entidades "Documentos de arquivo"
A entidade NEDA Agente possui mapeamento direto para a entidade RiC-E07 Agent. Os seus subtipos Instituição, Família e Persona, da mesma forma, possuem os seus correspondentes RiC-E11 Corporate Body, RiC-E10 Family e RiC-E08 Person, respectivamente. O subtipo RiC-E12 Position não possui entidade equivalente projetada no NEDA, no entanto, o Portal de Arquivos Espanhóis (Portal de Archivos Españoles - PARES) utiliza na prática uma entidade com características semelhantes para registrar a posição ou cargo de um agente. O subtipo RiC-E13 Mechanism não encontra correspondência no modelo NEDA.

O mapeamento da entidade NEDA Função e suas divisões deve ser feito através da entidade RiC-E14 Event e o seu subtipo RiC-E15 Activity. As entidades NEDA Função, Divisão de função e Ação correspondem à própria entidade RiC-E14 Event, enquanto a entidade NEDA Atividade/processo corresponde à entidade RiC-E15 Activity. O modelo NEDA torna clara a relação hierárquica das funções, o que não acontece no RiC, no entanto, é prevista a relação "RiC-R006 has sub event" a ser usada para compor arranjos de eventos e atividades.

A entidade NEDA Norma corresponde à entidade RiC-16 Rule podendo-se utilizar conforme o caso e, após uma análise criteriosa, a entidade RiC-17 Mandate.

Os lugares físicos representados pela entidade NEDA Lugar são mapeados diretamente para a entidade RiC-E22 Place. Enquanto no NEDA a entidade Lugar possui somente o tipo de relação "é assunto de", no RiC, a entidade RiC-E22 Place possui as relações "RiC-R074 is place associated", "RiC-R075 is location of", "RiC-R076 is jurisdiction of", "RiC-R007 contains", "RiC-R077 is adjacent to" e "RiC-R078 overlaps".

As demais abstrações do mundo real que necessitam ser descritas e não se encaixam nas definições das outras entidades devem utilizar a entidade NEDA "Conceito, objeto e acontecimento", podendo se relacionar normalmente com as demais entidades do modelo. Similarmente, no RiC, a entidade raiz RiC-E01 Thing pode ser utilizada para descrever conceitos que não se enquadram nos tipos das demais entidades, no entanto, a sua utilização desordenada pode tornar a interpretação das descrições difícil, assim como a migração para outros modelos conceituais.

Uma atenção especial foi dada às datas no RiC, dedicando quatro entidades para registrar esse tipo de informação, já no NEDA, as datas devem ser informadas nos atributos das entidades e relações, quando necessário. Do ponto de vista de 
representação da informação, projetar uma entidade específica para as datas e períodos permite que sejam feitas relações delas com as entidades, facilitando o entendimento global da descrição do arquivo. A forma como diversas universidades funcionam, através de semestres acadêmicos, se torna refletida com a utilização dessa entidade e suas relações com os documentos produzidos no decorrer desse período, por exemplo.

\section{Conclusões}

A utilização de padrões para a descrição arquivística visa o estabelecimento de um entendimento universal na comunidade assim como a normalização das informações inseridas. Os padrões internacionais buscam fornecer meios para que possa haver integração entre as plataformas de descrição e promover o intercâmbio de dados entre as instituições.

Os padrões nacionais, como é o caso do NEDA$M C$, visam adaptar a descrição para a realidade de cada país conforme os seus usos práticos. No entanto, para que as descrições possam navegar e se mesclar entre as instituições, é necessário haver um mapeamento desses modelos regionais para um modelo comum interpretável por outros países, como é o caso dos padrões internacionais.

O ICA, através do modelo conceitual e a ontologia do $\mathrm{RiC}$, pretende criar um ponto comum da descrição arquivística que possa ser usado globalmente e, na medida necessária, ser adaptado às realidades peculiares de cada instituição custodiadora de documentos. O processo de elaboração do modelo ainda está em andamento, pois prevê o recebimento de colaborações da comunidade arquivística. Assim mesmo, a realização de estudos das versões prévias deve ser realizada e incentivada a fim de embasar futuras mudanças no modelo.

Sabe-se que, após a finalização do modelo, novos estudos serão necessários para identificar as mudanças e adaptações necessárias tanto na metodologia de descrição quanto nas ferramentas de descrição. Da mesma forma, a migração das descrições existentes, deve ser realizada cuidadosamente para que não hajam erros ou perda dos dados, envolvendo, sempre que possível, equipes de arquivistas e profissionais dos sistemas gerenciadores de documentos. $\mathrm{O}$ presente estudo pode ainda auxiliar a guiar os trabaIhos futuros de migração para o RiC dos padrões nacionais e regionais que têm as normas ISAD(G), ISAAR(CPF), ISDF e ISDIAH como pedra basilar, auxiliando na compreensão dos conceitos envolvidos.

\section{Referências}

Archivo General de la Nación (República Dominicana) (2019). NORDA: Norma Dominicana de Descripción Archivística. Santo Domingo, República Dominicana.

Archivo General de la Nación (Uruguai) (2016). NUDA: Norma Uruguaya de Descripción Archivística. Montevideo, Uruguai.

CNEDA. Comisión de Normas Españolas de Descripción Archivística (2017). NEDA-MC: modelo conceptual de descripción archivística: entidades, relaciones y atributos.

Conselho Nacional de Arquivos (2016). RIC-CM: consultation draft v0.1. Comments by Brazilian Rio de Janeiro Group and Câmara Técnica de Documentos Eletrônicos of Conselho Nacional de Arquivos. (Dezembro, 2016).

Conselho Nacional de Arquivos (2006). NOBRADE: Norma Brasileira de Descrição Arquivística. Rio de Janeiro, Brasil. http://www.siga.arquivonacional.gov.br/images/public acoes/nobrade.pdf (2020-04-20).

Generalitat de Cataluña (2007). NODAC: Norma de Descripción Archivística de Cataluña. Barcelona, Espanha.

Gillean, Dan (2017). Artefactual response to RiC-CM Draft. https://groups.google.com/d/msg/ica-atom-users/QwSor 7OQ90U/DpsDM1P5BAAJ (2020-03-18).

Grupo de Arquiveiros de Galícia (2010). NOGADA: Norma Galega de Descrición Arquivística.

Interpares Trust (2016). InterPARES Trust response to EGAD-RiC. https://interparestrust.org/trust/article/interpa res-trust-response-to-egadric (2020-03-18).

Llanes-Padrón, Dunia (2011). La representación nornalizada de los documentos. Estudio comparado de normas de descripción. Tesis doctoral (Doctorado en Biblioteconomía y Documentación). Salamanca: Universidad de Salamanca, Julio, 2011. 766 p.

Llanes-Padrón, Dunia; Moro-Cabero, Manuela (2017). Records in contexts: Un nuevo modelo para la representación de la información archivística en el entorno de la web semántica. // El Profesional de la Información. 26: 3 (Junio, 2017) 525-533.

ICA. International Council on Archives (2000). ISAD(G): General International Standard Archival Description: adopted by the Committee on Descriptive Standards, Stockholm, Sweden, 19-22 September 1999. 2nd ed. Ottawa: International Council on Archives, 2000.

ICA. International Council on Archives (2004). ISAAR (CPF): International Sstandard Archival Authority Record for Corporate Bodies, Persons and Families. 2nd. ed. Canberra, Australia: Committee on Descriptive Standards, 2004.

ICA. International Council on Archives (2007). ISDF: International Standard for Describing Functions. 1st. ed. Desden, Germany: Committee on Best Practices and Standards, 2007

ICA. International Council on Archives (2008). ISDIAH: International Standard for Describing Institutions with Archival Holdings. 1st. ed. London, United Kingdom: Committee on Best Practices and Standards, 2008.

ICA. International Council on Archives (2016). Records In Context: a Conceptual Model for Archival Description. Consultation Draft v0.1 ed. Experts Group on Archival Description, 2016.

ICA. International Council on Archives (2019). Records In Context: a Conceptual Model for Archival Description. Consultation Draft v0.2 (preview). Experts Group on Archival Description, 2019. https://www.ica.org/sites/defa ult/files/ric-cm-0.2_preview.pdf (2020-03-06).

ICA. International Council on Archives (2019). Records In Context Ontology. V0.1. ed, 2019. https://www.ica.org /standards/RiC/ontology.html (2020-03-06). 
ICA. International Council on Archives. Records In Context Ontology. 2019. https://www.ica.org/en/records-in-contexts-ontology. (2020-03-06).

Snyder, Alan (1986). Encapsulation and inheritance in objectoriented programming languages. // Conference proceedings on Object-oriented programming systems, languages and applications (OOPSLA '86). New York, NY, USA: Association for Computing Machinery. 38-45. https://doi.org/10.1145/28697.28702 (2020-03-12).
Requejo Zalama, Javier (2018). Documentos em contexto: las nuevas normas de descripción archivística y su aplicación en la administración digital. // El archivo electrónico en la administración digital: 23 Jornadas de Archivos Universitarios, 21-23 de junio de 2017, A Coruña. 27-4). http://hdl.handle.net/2183/20675 (2020-05-19).

Enviado: 2020-04-28. Segunda versión: 2020-10-05. Aceptado: 2020-11-18. 